\title{
A Service-based Congestion Control Strategy for Content Centric Networking
}

\author{
Zhang Chengchen,Wang Lei ${ }^{1, a}$,Wang Zhenfa,Wang Song \\ ${ }^{1}$ Department of Automation,University of Science and Technology of \\ China, \\ Hefei Anhui 230027,China \\ awangl@ustc.edu.cn
}

\begin{abstract}
Congestion control for Content Centric Networking is one of the hottest research point.Existing solutions mostly reference TCP congestion control.Most solutions didn’t distinguish the traffic classes, which reduce some services’ QoS.In order to address this problem,we propose in this paper a service-based congestion control strategy for CCN.We design the algorithm to choose service requester to feedback the congestion level of the network by monitoring queue of Interests in the CCN router.Our evaluation shows that our strategy can effectively increase QoS.
\end{abstract}

Keywords:Content Centric Networking;Congestion Control;Service-Based

\section{Introduction}

In 2009,Van Jacobson and the PARC research group proposed the CCN[1] framework, and then NDN project started based on CCN. CCN retains the simplicity and scalability and offer much better disruption tolerance,delivery efficiency and security[1]. Although CCN has many advantages,there are still some problems to be studied.CCN designs a simple flow control mechanism,namely one Interest retrieves at most one Data packet.But this 
mechanism cannot be effective,also may be not very fair.At present,many people have proposed solutions to this problem.

ICP[2] and ICTP[3] are receiver-driven solutions which use congestion strategies are similar to TCP.They calculated an Interest retransmission timer from previous RTT samples of Data packets,regardless of origin.HoBHIS[4] and HR-ICP[5] need maintain large amount of state information to predict the congestion,which affect the scalability of the network .Since content chunks in CCN may be retrieved from different nodes/caches,CСТCP[6] proposed a novel anticipated Interests mechanism to predict the location of chunks before they are requested.Although CCTCP has solved the problem of RTT unpredictability,the mechanism brings much overhead to the system.ECA[7] presents an explicit feedback congestion control algorithm.It proactive detect the length of transmission queue in the CCN router to get the congestion level of network and explicitly feedback to the receiver.

All these studies control Interest rate without consideration of specific service,which will affect the QoS.In order to solve this problem,this paper proposes a service-based congestion control strategy for content centric networking.By monitoring the queue of Interests in the CCN router,the router feedback to different service requesters according to congestion level.

\section{Model Design}

Although the concept of Data flowing along an end-to-end path does not apply, the possibility that the chunks from the same content requested by a receiver will pass the same CCN router is very large.The paper is based on above assumptions.In the design of this paper,each Interest packet will be added two fields,content type and priority.The content type is used to distinguish between different services.According to the division of network services by 3GPP[8],services are divided into four classes,Conversation class,Streaming class,Interactive class and Background class.From Conversation class to Background class,their delay requirements gradually decrease,but their 
requirements for packet loss rate increase.Priority is used to distinguish the grade of service.

In this paper,we check the state of network by monitoring the queue of Interest packets in the CCN router.Once congestion happens,the feedback information will be transferred to the corresponding receiver.Then the receiver will regulate Interest rate,so as to achieve the purpose of congestion control.The congestion model is shown in Figure 1.Intermediate routers receive multiple Interest packets from requesters and they will be sent to the same export link.

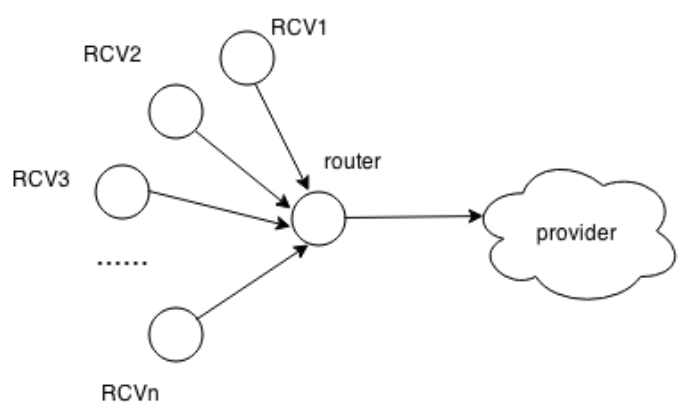

Fig.1. The congestion model

By calculating sum of weighted numbers,we can get a value of congestion degree.When the value is bigger than the congestion threshold,the congestion may occurs and the congestion processing should be done.The value of congestion degree is calculated by the following formula:

$$
V=w_{c} * n_{c}+w_{s}^{*} n_{s}+w_{i}^{*} n_{i}+w_{b} * n_{b}
$$

where $\mathrm{V}$ is for the value of congestion degree; $n_{c}, n_{s}, n_{i}, n_{b}, w_{c}, w_{s}, w_{i}, w_{b}$ are the numbers and weights of services from Conversation class to Background class and they meet:

$0<w_{c}<w_{s}<w_{i}<w_{b}$

(2)

According to the approach in the [7],we divide the congestion level into three categories.When the congestion degree is smaller than $V_{\text {busy }}$,the state of link is 
idle.When the congestion degree is greater than $V_{\text {congestion }}$,the state of link is congestion.Otherwise the state of link is busy.

\section{Algorithm Description}

As mentioned above,after discovery of congestion,the service that have the lowest requirement for delay would be processed firstly.Thus we can ensure the quality of the majority of services as much as possible.Then the CCN router will check for the congestion level at certain intervals. The following is a description of the algorithm.

Table 1. Select-Interest Algorithm 
Interest rate to adjust to the state of network.For the rate regulation,we adopt the approach proposed in [7].When the congestion happens,the Interest window is multiplied by a coefficient $\alpha$ which is smaller than 1 ; when the state is busy,the Interest window plus an increment $\beta$;otherwise the Interest window multiplied by a coefficient $\gamma$ which is bigger than 1 .

\section{Performance evaluation}

To demonstrate the effectiveness of our congestion control strategy, this paper simulates the real environment of CCN using ndnSIM, and makes a series of simulation experiments. We implement it on a PC with operation system of Ubuntu 14.04,a 2.30GHz CPU and 4.0GB RAM.During the experiments, we use different types of services to test the finish time of whole services. The experimental results are compared with ECA and AIMD, the former is an explicit control algorithm and the latter is a strategy commonly used by existing transmission protocols and congestion control algorithm.To simulate the situation of congestion, we use a topology of Figure 1, various requests being destined for the same link.

Table 2. Situations in experiment

\begin{tabular}{|c|c|}
\hline $\begin{array}{l}\text { S } \\
\text { ituati } \\
\text { on1 }\end{array}$ & $\begin{array}{l}\mathrm{S} 1(3,1,150,15), \mathrm{S} 2(3,2,300,15), \mathrm{S} 3(3,3,225,15), \mathrm{S} 4(4,1,200,20), \mathrm{S} 5(4,2 \text {, } \\
300,20), \mathrm{S} 6(4,3,200,20)\end{array}$ \\
\hline $\begin{array}{l}\text { S } \\
\text { ituati } \\
\text { on2 }\end{array}$ & $\begin{array}{l}\mathrm{S} 1(1,2,100,5), \mathrm{S} 2(2,1,100,10), \mathrm{S} 3(2,2,150,10), \mathrm{S} 4(2,2,200,10), \mathrm{S} 5(3,1,1 \\
50,15), \mathrm{S} 6(3,2,300,15), \mathrm{S} 7(4,1,200,20), \mathrm{S} 8(4,2,200,20)\end{array}$ \\
\hline $\begin{array}{l}\text { S } \\
\text { ituati } \\
\text { on3 }\end{array}$ & $\begin{array}{l}\text { S1(1,1,50,5),S2(1,2,75,5),S3(1,2,100,5),S4(2,1,100,10),S5(2,2,150,1 } \\
\text { 0),S6(2,2,200,10),S7(3,1,150,15),S8(3,2,150,15),S9(4,1,200,20), } \\
\text { S10 }(4,2,200,20)\end{array}$ \\
\hline \multicolumn{2}{|r|}{$\begin{array}{l}\text { Our experiment simulates three situations of congestion, as shown in Table } 2 \text {. } \\
\text { ch service listed in the table has four parameters. The first parameter } \\
\text { resents the type of service, 1-4 respectively representing services from } \\
\text { nversation class to Background class; the second indicates the priority, the } \\
\text { ger the number, the higher the priority; the third indicates the amount of Data; } \\
\text { fourth indicates the bandwidth of Data packet corresponding to the initial } \\
\text { nsmission rate of Interest packet set by users. Take situation } 1 \text { for example. S1 }\end{array}$} \\
\hline
\end{tabular}


$(3,1,150,15)$ indicates that this is an interactive class of service, the priority level is 1 , the size of requested Data is $150 \mathrm{MB}$, and the initial Data packet bandwidth is $15 \mathrm{Mbps}$.The link outlet bandwidth is set to $100 \mathrm{Mbps}$ during the experiment.

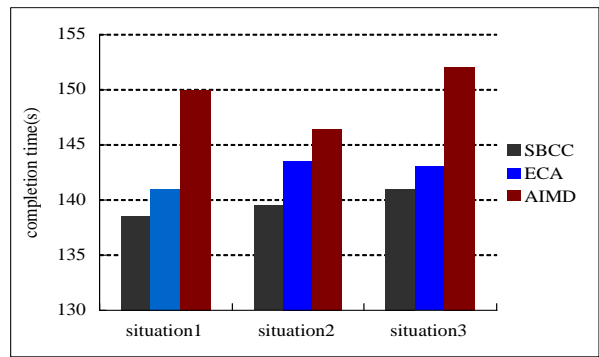

Fig.2. service completion time

Figure 2 is the experimental results of Table 2, we can see that the SBCC (service-based congestion control) costs the shortest time to complete all the services, followed by the ECA, and the worst of the three strategies is AIMD. The main reason why SBCC gets better performance than is that it didn't adopt the ECA's initially reducing all Interest packets' rate regardless of service. In contrast, SBCC regulate the Interest rate from the services of background class which is the most delay-insensitive compared with the other classes. Our experimental result shows that the SBCC can improve overall completion time of services.

\section{Conclusion}

There are many research achievements on the network congestion control of CCN, but they didn't take service factor into account, which is likely to affect the QoS. To solve this problem, we propose a service-based congestion control (SBCC) strategy. By checking the queue of Interest packets which are waiting to be sent in CCN router, we detect congestion degree of network. Once congestion happens, SBCC first regulate the Interest rate of the service with the lowest priority which belongs to the most latency insensitive classes of services Our experiments shows that SBCC can reduce the completion time of services and effectively improve the QoS. 
There are some deficiencies need to be further improved in future work in this paper. Firstly, the experiments conducted in this paper base on a relatively static scene, but the actual network is subject to a variety of changing services. Secondly, there is no consideration of multiple requesters requesting the same service, and the priority of popular content should be improved. All above will be our directions of future work.

\section{Acknowledgement}

In this paper, the research was supported by "the Fundamental Research Funds for the Central Universities" (Grant No. WK2100100012) and the "Strategic Priority Research Program" of the Chinese Academy of Sciences(Grant No. XDA06030900).

\section{References}

[1]Jacobson V, Smetters D K, Thornton J D, et al. Networking named content[C]/Proceedings of the 5th international conference on Emerging networking experiments and technologies. ACM, 2009: 1-12.

[2]Carofiglio G, Gallo M, Muscariello L. ICP: Design and evaluation of an Interest control protocol for content-centric networking[C]//Computer Communications Workshops (INFOCOM WKSHPS), 2012 IEEE Conference on. IEEE, 2012: 304-309.

[3]Salsano S, Detti A, Cancellieri M, et al. Transport-layer issues in information centric networks[C]/Proceedings of the second edition of the ICN workshop on Information-centric networking. ACM, 2012: 19-24.

[4]Rozhnova N, Fdida S. An effective hop-by-hop Interest shaping mechanism for CCN communications[C]//Computer Communications Workshops (INFOCOM WKSHPS), 2012 IEEE Conference on. IEEE, 2012: 322-327.

[5]Carofiglio G, Gallo M, Muscariello L. Joint hop-by-hop and receiver-driven Interest control protocol for content-centric networks[C]//Proceedings of the second edition of the ICN workshop on Information-centric networking. ACM, 2012: 37-42.

[6]Saino L, Cocora C, Pavlou G. Cctcp: A scalable receiver-driven congestion 
control protocol for content centric networking[C]//Communications (ICC), 2013 IEEE International Conference on. IEEE, 2013: 3775-3780.

[7]Tang Xiao,Ren Yongmao,Li Jin et al.A Congestion Control Algorithm Based on Explicit Feedback for NDN[J].E-science Technology \& Application, 2014, 3 : 009.

[8]Quality of Service (QoS) concept and architecture.http://www.3gpp.org/DynaReport/23107.htm 